\title{
Three periodic solutions for a class of higher-dimensional functional differential equations with impulses
}

\author{
by Yongkun Li, Changzhao Li and Juan Zhang (Kunming)
}

\begin{abstract}
By using the well-known Leggett-Williams multiple fixed point theorem for cones, some new criteria are established for the existence of three positive periodic solutions for a class of $n$-dimensional functional differential equations with impulses of the form

$$
\left\{\begin{array}{l}
y^{\prime}(t)=A(t) y(t)+g\left(t, y_{t}\right), \quad t \neq t_{j}, j \in \mathbb{Z}, \\
y\left(t_{j}^{+}\right)=y\left(t_{j}^{-}\right)+I_{j}\left(y\left(t_{j}\right)\right),
\end{array}\right.
$$
\end{abstract}

where $A(t)=\left(a_{i j}(t)\right)_{n \times n}$ is a nonsingular matrix with continuous real-valued entries.

1. Introduction. Impulsive delay differential equations may express several real-world simulation processes which depend on their prehistory and are subject to short time disturbances. Such processes occur in the theory of optimal control, population dynamics, biotechnologies, economics, etc. In recent years, the existence of positive periodic solutions of delay differential equations with impulsive effects has been an object of active research; we refer the reader to $[4-7,10-15]$.

Recently, based on a fixed point theorem in cones, Li et al. ([5]) investigated the periodicity of the following scalar system:

$$
\left\{\begin{array}{l}
\dot{y}(t)=-a(t) y(t)+g\left(t, y(t-\tau(t)), \quad t \neq t_{j}, j \in \mathbb{Z},\right. \\
y\left(t_{j}^{+}\right)=y\left(t_{j}^{-}\right)+I_{j}\left(y\left(t_{j}\right)\right),
\end{array}\right.
$$

where $a \in C(\mathbb{R},(0, \infty)), \tau \in C(\mathbb{R}, \mathbb{R}), g \in C(\mathbb{R} \times[0, \infty),[0, \infty)), a, \tau$ are $\omega$-periodic functions and $g$ is $\omega$-periodic with respect to its first argument. It is well known that system (1.1) includes many mathematical ecological models (see $[2,6]$ ).

Also, in [2], Jiang et al. employed Krasnosel'skiil's fixed point theorem for cones to study the existence of positive periodic solutions of the system

2010 Mathematics Subject Classification: Primary 34K13; Secondary 34K32.

Key words and phrases: positive periodic solutions, functional differential equations, impulse, Leggett-Williams fixed point theorem. 
of functional differential equations

$$
\dot{x}(t)=A(t) x(t)+f\left(t, x_{t}\right),
$$

where $A(t)=\operatorname{diag}\left[a_{1}(t), \ldots, a_{n}(t)\right], a_{j} \in C(\mathbb{R}, \mathbb{R})$ is $\omega$-periodic, $f$ is a function defined on $\mathbb{R} \times B C\left(\mathbb{R}, \mathbb{R}^{n}\right)$, and $f\left(t, x_{t}\right)$ is $\omega$-periodic whenever $x$ is $\omega$-periodic, where $B C\left(\mathbb{R}, \mathbb{R}^{n}\right)$ denotes the Banach space of bounded continuous functions $\phi: \mathbb{R} \rightarrow \mathbb{R}^{n}$ with the norm $\|\phi\|=\sup _{\theta \in \mathbb{R}} \sum_{j=1}^{n}\left|\phi_{j}(\theta)\right|$ where $\phi=\left(\phi_{1}, \ldots, \phi_{n}\right)^{T}$, and $\omega>0$ is a constant. If $x \in B C\left(\mathbb{R}, \mathbb{R}^{n}\right)$, then $x_{t} \in B C\left(\mathbb{R}, \mathbb{R}^{n}\right)$ for $t \in \mathbb{R}$ is defined by $x_{t}(\theta)=x(t+\theta)$ for $\theta \in \mathbb{R}$.

However, to the best of our knowledge, there are few papers published on the multiple existence of positive periodic solutions for higher-dimensional functional differential equations with impulses; moreover, the existing results on the existence of periodic solutions for system (1.2) with or without impulses all assume that the coefficient matrix $A(t)$ is diagonal. Motivated by the above, in this paper, we are concerned with the following system:

$$
\left\{\begin{array}{l}
y^{\prime}(t)=A(t) y(t)+g\left(t, y_{t}\right), \quad t \neq t_{j}, j \in \mathbb{Z}, \\
y\left(t_{j}^{+}\right)=y\left(t_{j}^{-}\right)+I_{j}\left(y\left(t_{j}\right)\right),
\end{array}\right.
$$

where $A(t)=\left(a_{i j}(t)\right)_{n \times n}$ is a nonsingular matrix with continuous real-valued functions as entries and $A(t+T)=A(t) ; g=\left(g_{1}, \ldots, g_{n}\right)^{T}$ is a functional $\mathbb{R} \times B C\left(\mathbb{R}, \mathbb{R}^{n}\right) \rightarrow \mathbb{R}^{n}$, satisfying $g\left(t+T, y_{t+T}\right)=g\left(t, y_{t}\right)$ for all $t \in \mathbb{R}, y_{t} \in$ $B C\left(\mathbb{R}, \mathbb{R}^{n}\right)$, and if $y \in B C\left(\mathbb{R}, \mathbb{R}^{n}\right)$ then for any $t \in \mathbb{R}, y_{t} \in B C\left(\mathbb{R}, \mathbb{R}^{n}\right)$ is defined by $y_{t}(s)=y(t+s)$ for $s \in \mathbb{R} ; y\left(t_{j}^{+}\right), y\left(t_{j}^{-}\right)=y\left(t_{j}\right)$ represent the right and the left limit of $y(t)$ at $t_{j}, j \in \mathbb{Z}$, respectively; and $I_{j}=$ $\left(I_{j}^{1}, I_{j}^{2}, \ldots, I_{j}^{n}\right)^{T} \in C\left(\mathbb{R}^{n}, \mathbb{R}^{n}\right), j \in \mathbb{Z}$. We assume that there exists an integer $p>0$ such that $t_{j+p}=t_{j}+T, I_{j+p}=I_{j}, j \in \mathbb{Z}$, where $0<t_{1}<\cdots<t_{p}<T$.

The Leggett-Williams multiple fixed point theorem [3] has proved to be a successful technique for dealing with the existence of three positive solutions of two, three or multi-point boundary value problems for differential equations (see $[8,9,11]$ ). Our main aim is (by using the Leggett-Williams theorem) to study the existence of at least three positive periodic solutions of (1.3). In the analysis we use the fundamental solution matrix of

$$
y^{\prime}=A(t) y
$$

and convert system (1.3) into an integral equation. Then we employ the Leggett-Williams theorem to show the existence of triple positive periodic solutions of (1.3). Our methods are different from those used in [2, 5, 8, 9].

In this paper, for each $x=\left(x_{1}, \ldots, x_{n}\right)^{T} \in \mathbb{R}^{n}$, the norm of $x$ is defined as $|x|_{0}=\sum_{i=1}^{n}\left|x_{i}\right|$. For matrices $A, B$, the notation $A>B(A \leq B)$ means that each pair of corresponding entries of $A$ and $B$ satisfies the inequality ">" (" $\leq$ "). In particular, $A$ is called a positive matrix if $A>0$.

In what follows, we assume that 
$\left(H_{1}\right) g\left(t, \varphi_{t}\right)$ is a continuous functional of $t$ for each $\varphi \in B C\left(\mathbb{R}, \mathbb{R}^{n}\right)$.

$\left(H_{2}\right)$ For any $L>0$ and $\varepsilon>0$, there exists $\delta>0$ such that $[\varphi, \psi \in$ $B C\left(\mathbb{R}, \mathbb{R}^{n}\right),\|\varphi\| \leq L,\|\psi\| \leq L$ and $\left.\|\varphi-\psi\| \leq \delta\right]$ implies that

$$
\left|g\left(t, \varphi_{t}\right)-g\left(t, \psi_{t}\right)\right|_{0}<\varepsilon, \quad \forall t \in[0, T] .
$$

To simplify our description, we introduce the following notations:

$$
\begin{aligned}
g^{\alpha} & =\limsup _{\|y\| \rightarrow \alpha} \frac{\int_{0}^{T}\left|g\left(s, y_{s}\right)\right|_{0} d s}{\|y\|}, & I^{\alpha}=\limsup _{\|y\| \rightarrow \alpha} \frac{\sum_{j=1}^{p}\left|I_{j}(y)\right|_{0}}{\|y\|}, \\
\check{g}=\inf _{\delta b \leq u \leq b} \int_{0}^{T}|g(s, u)|_{0} d s, & \check{I} & =\inf _{\delta b \leq u \leq b} \sum_{j=1}^{p}\left|I_{j}(u)\right|_{0} .
\end{aligned}
$$

The organization of the rest of this paper is as follows: In Section 2, we introduce some notations and definitions, and state some preliminary results. In Section 3, by using the Leggett-Williams multiple fixed point theorem, we establish the existence of at least three positive periodic solutions of (1.3).

2. Preliminaries. For convenience, we first recall the related definitions and the fixed point theorem which will be used to prove our main results.

Definition 2.1. Let $X$ be a Banach space and $K$ be a closed, nonempty subset of $X$. Then $K$ is a cone if

(i) $\alpha u+\beta v \in K$ for all $u, v \in K$ and all $\alpha, \beta \geq 0$;

(ii) $u,-u \in K$ imply $u=0$.

Every cone $K \subset X$ includes an ordering in $X$ given by $x \leq y$ if and only if $y-x \in K$.

Define $K_{r}=\{x \in K:\|x\| \leq r\}$. Let $\alpha$ denote a positive continuous concave functional on $K$, that is, $\alpha: K \rightarrow[0, \infty)$ is continuous and satisfies

$$
\alpha(\lambda x+(1-\lambda) y) \geq \lambda \alpha(x)+(1-\lambda) \alpha(y), \quad \forall x, y \in K, 0 \leq \lambda \leq 1,
$$

and we set $K(\alpha, a, b)=\{x \in K: a \leq \alpha(x),\|x\| \leq b\}$.

The following lemma from [3] is useful for the proof of our main results.

Lemma 2.1 (Leggett-Williams [3]). Let $K$ be a cone of the real Banach space $X$ and $H: \bar{K}_{c} \rightarrow \bar{K}_{c}$ be a completely continuous operator, and suppose that there exist a concave positive functional $\alpha$ with $\alpha(x) \leq\|x\|(x \in K)$ and numbers $a, b, d$ with $0<d<a<b \leq c$, satisfying the following conditions:

(i) $\{x \in K(\alpha, a, b): \alpha(x)>a\} \neq \emptyset$ and $\alpha(H x)>a$ if $x \in K(\alpha, a, b)$;

(ii) $\|H x\|<d$ if $x \in K_{d}$;

(iii) $\alpha(H x)>a$ for all $x \in K(\alpha, a, c)$ with $\|H x\|>b$.

Then $H$ has at least three fixed points $x_{1}, x_{2}, x_{3} \in \bar{K}_{c}$ such that

$$
x_{1} \in K_{d}, x_{2} \in\{x \in K(\alpha, a, c): \alpha(x)>a\}, x_{3} \in \bar{K}_{c} \backslash \alpha\left(K(\alpha, a, c) \cup \bar{K}_{d}\right) .
$$


Definition $2.2([1,12])$. If the matrix $A(t)$ is $T$-periodic, then the linear system

$$
y^{\prime}=A(t) y
$$

is said to be noncritical with respect to $T$ if it has no periodic solution of period $T$ except the trivial solution $y=0$.

Throughout this paper it is assumed that system (1.4) is noncritical. Let $\Phi(t)$ be the fundamental solution matrix of (1.4) with $\Phi(0)=I$, where $I$ is the $n \times n$ identity matrix. Then we have the following results $[1,12]$.

(i) $\operatorname{det} \Phi(t) \neq 0$;

(ii) there exists a constant matrix $B$ such that $\Phi(t+T)=\Phi(t) e^{B T}$, by Floquet theory;

(iii) system (1.4) is noncritical if and only if $\operatorname{det}(I-\Phi(T)) \neq 0$.

Lemma 2.2. Let $\Phi(t)$ be the fundamental solution matrix of (1.4) with $\Phi(0)=I$. Then

$$
\Phi(t)\left[(\Phi(t)-\Phi(t+T))^{-1}-(\Phi(t-T)-\Phi(t))^{-1}\right]=I .
$$

Proof. We have

$$
\begin{aligned}
\Phi(t)[( & \left.\Phi(t)-\Phi(t+T))^{-1}-(\Phi(t-T)-\Phi(t))^{-1}\right] \\
& =\left[(\Phi(t)-\Phi(t+T)) \Phi^{-1}(t)\right]^{-1}-\left[(\Phi(t-T)-\Phi(t)) \Phi^{-1}(t)\right]^{-1} \\
& =\left(I-\Phi(t+T) \Phi^{-1}(t)\right)^{-1}-\left(\Phi(t-T) \Phi^{-1}(t)-I\right)^{-1} \\
& =\left(I-\Phi(t) e^{B T} \Phi^{-1}(t)\right)^{-1}-\left(\Phi(t) e^{-B T} \Phi^{-1}(t)-I\right)^{-1} \\
& =\Phi(t) e^{-B T} \Phi^{-1}(t)\left(\Phi(t) e^{-B T} \Phi^{-1}(t)-I\right)^{-1}-\left(\Phi(t) e^{-B T} \Phi^{-1}(t)-I\right)^{-1} \\
& =\left(\Phi(t) e^{-B T} \Phi^{-1}(t)-I\right)\left(\Phi(t) e^{-B T} \Phi^{-1}(t)-I\right)^{-1}=I .
\end{aligned}
$$

In order to obtain the existence of periodic solutions of system (1.3), we make the following preparations.

Define

$$
\begin{array}{r}
P C\left(\mathbb{R}, \mathbb{R}^{n}\right)=\left\{y: \mathbb{R} \rightarrow \mathbb{R}^{n}\left|y_{i}\right|_{\left(t_{j}, t_{j+1}\right)} \in C\left(t_{j}, t_{j+1}\right), \exists y\left(t_{j}^{-}\right)=y\left(t_{j}\right), y\left(t_{j}^{+}\right),\right. \\
j \in \mathbb{Z}, i=1, \ldots, n\}
\end{array}
$$

and set

$$
X=\left\{y \in P C\left(\mathbb{R}, \mathbb{R}^{n}\right): y(t+T)=y(t) \text { for all } t\right\}
$$

with the norm defined by $\|y\|=\sup _{t \in \mathbb{R}}|y(t)|_{0}=\sup _{t \in[0, T]}|y(t)|_{0}$, where $|y(t)|_{0}=\sum_{i=1}^{n}\left|y_{i}(t)\right|$. Then $X$ is a Banach space.

The following lemma is fundamental to our discussion:

LEMMA 2.3. A function $y$ is a T-periodic solution of (1.3) if and only if $y$ is a T-periodic solution of the integral equation

$$
y(t)=\int_{t}^{t+T} G(t, r) g\left(r, y_{r}\right) d r+\sum_{j: t_{j} \in[t, t+T)} G\left(t, t_{j}\right) I_{j}\left(y\left(t_{j}\right)\right),
$$


where

$$
G(t, u)=\Phi(t)(\Phi(u-T)-\Phi(u))^{-1}:=\left(G_{i k}\right)_{n \times n}, \quad u \in[t, t+T) .
$$

Proof. If $y$ is a $T$-periodic solution of (1.3), then for any $t \in \mathbb{R}$, there exists $j \in \mathbb{Z}$ such that $t_{j}$ is the first impulsive point after $t$. Let $\Phi(t)$ be a fundamental solution of system (1.4). Since $\Phi(t) \Phi^{-1}(t)=I$, it follows that

$$
\begin{aligned}
0 & =\frac{d}{d t}\left(\Phi(t) \Phi^{-1}(t)\right)=\frac{d}{d t}(\Phi(t)) \Phi^{-1}(t)+\Phi(t) \frac{d}{d t}\left(\Phi^{-1}(t)\right) \\
& =(A(t) \Phi(t)) \Phi^{-1}(t)+\Phi(t) \frac{d}{d t}\left(\Phi^{-1}(t)\right)=A(t)+\Phi(t) \frac{d}{d t}\left(\Phi^{-1}(t)\right) .
\end{aligned}
$$

This implies that

$$
\frac{d}{d t}\left(\Phi^{-1}(t)\right)=-\Phi^{-1}(t) A(t)
$$

By (2.4), we have

$$
\begin{aligned}
\frac{d}{d t}\left(\Phi^{-1}(t) y(t)\right) & =\frac{d}{d t}\left(\Phi^{-1}(t)\right) y(t)+\Phi^{-1}(t) \frac{d}{d t}(y(t)) \\
& =-\Phi^{-1}(t) A(t) y(t)+\Phi^{-1}(t)\left[A(t) y(t)+g\left(t, y_{t}\right)\right] \\
& =\Phi^{-1}(t) g\left(t, y_{t}\right)
\end{aligned}
$$

An integration of (2.5) from $t$ to $s$ for $s \in\left[t, t_{j}\right], j \in \mathbb{Z}$, yields

$$
y(s)=\Phi(s) \int_{t}^{s}\left[\Phi^{-1}(r) g\left(r, y_{r}\right)\right] d r+\Phi(s) \Phi^{-1}(t) y(t),
$$

so

$$
y\left(t_{j}\right)=\Phi\left(t_{j}\right) \int_{t}^{t_{j}}\left[\Phi^{-1}(r) g\left(r, y_{r}\right)\right] d r+\Phi\left(t_{j}\right) \Phi^{-1}(t) y(t), \quad j \in \mathbb{Z} .
$$

Again, integrating (2.5) over $\left(t_{j}, t_{j+1}\right], j \in \mathbb{Z}$, we get

$$
\begin{aligned}
y(s)= & \Phi(s) \Phi^{-1}\left(t_{j}\right) y\left(t_{j}^{+}\right)+\int_{t_{j}}^{s} \Phi(s) \Phi^{-1}(r) g\left(r, y_{r}\right) d r \\
= & \Phi(s) \Phi^{-1}\left(t_{j}\right)\left[y\left(t_{j}^{-}\right)+I_{j}\left(y\left(t_{j}\right)\right)\right]+\int_{t_{j}}^{s} \Phi(s) \Phi^{-1}(r) g\left(r, y_{r}\right) d r \\
= & \Phi(s) \Phi^{-1}(t) y(t)+\Phi(s)\left[\int_{t}^{t_{j}} \Phi^{-1}(r) g\left(r, y_{r}\right) d r+\int_{t_{j}}^{s} \Phi^{-1}(r) g\left(r, y_{r}\right) d r\right] \\
& +\Phi(s) \Phi^{-1}\left(t_{j}\right) I_{j}\left(y\left(t_{j}\right)\right) \\
= & \Phi(s) \Phi^{-1}(t) y(t)+\Phi(s) \int_{t}^{s} \Phi^{-1}(r) g\left(r, y_{r}\right) d r+\Phi(s) \Phi^{-1}\left(t_{j}\right) I_{j}\left(y\left(t_{j}\right)\right) .
\end{aligned}
$$


Repeating the above process for $s \in[t, t+T]$, we obtain

$$
\begin{aligned}
y(s)= & \Phi(s) \Phi^{-1}(t) y(t)+\Phi(s) \int_{t}^{s} \Phi^{-1}(r) g\left(r, y_{r}\right) d r \\
& +\sum_{j: t_{j} \in[t, s)} \Phi(s) \Phi^{-1}\left(t_{j}\right) I_{j}\left(y\left(t_{j}\right)\right) .
\end{aligned}
$$

Let $s=t+T$ in the above equality. Then

$$
\begin{aligned}
y(t+T)= & \Phi(t+T) \Phi^{-1}(t) y(t)+\Phi(t+T) \int_{t}^{t+T} \Phi^{-1}(r) g\left(r, y_{r}\right) d r \\
& +\sum_{j: t_{j} \in[t, t+T)} \Phi(t+T) \Phi^{-1}\left(t_{j}\right) I_{j}\left(y\left(t_{j}\right)\right) .
\end{aligned}
$$

It follows from $y(t+T)=y(t)$ that

$$
\begin{aligned}
& \left(I-\Phi(t+T) \Phi^{-1}(t)\right) y(t) \\
& \quad=\Phi(t+T) \int_{t}^{t+T} \Phi^{-1}(r) g\left(r, y_{r}\right) d r+\Phi(t+T) \sum_{j: t_{j} \in[t, t+T)} \Phi^{-1}\left(t_{j}\right) I_{j}\left(y\left(t_{j}\right)\right) .
\end{aligned}
$$

So we get

$$
\begin{aligned}
y(t)= & \left(I-\Phi(t+T) \Phi^{-1}(t)\right)^{-1} \Phi(t+T)\left[\int_{t}^{t+T} \Phi^{-1}(r) g\left(r, y_{r}\right) d r\right. \\
& \left.+\sum_{j: t_{j} \in[t, t+T)} \Phi^{-1}\left(t_{j}\right) I_{j}\left(y\left(t_{j}\right)\right)\right] \\
= & \left(\Phi^{-1}(t+T)-\Phi^{-1}(t)\right)^{-1}\left[\int_{t}^{t+T} \Phi^{-1}(r) g\left(r, y_{r}\right) d r\right. \\
& \left.+\sum_{j: t_{j} \in[t, t+T)} \Phi^{-1}\left(t_{j}\right) I_{j}\left(y\left(t_{j}\right)\right)\right] \\
= & \Phi(t)\left(e^{-B T}-I\right)^{-1}\left[\int_{t}^{t+T} \Phi^{-1}(r) g(r, y(r), y(r-\tau(r))) d r\right. \\
& \left.+\sum_{j: t_{j} \in[t, t+T)} \Phi^{-1}\left(t_{j}\right) I_{j}\left(y\left(t_{j}\right)\right)\right] \\
= & \int_{t}^{t+T} \Phi_{(t)\left(e^{-B T}-I\right)^{-1} \Phi^{-1}(r) g\left(r, y_{r}\right) d r} \Phi_{(t)\left(e^{-B T}-I\right)^{-1} \Phi^{-1}\left(t_{j}\right) I_{j}\left(y\left(t_{j}\right)\right)}
\end{aligned}
$$




$$
\begin{aligned}
= & \int_{t}^{t+T} \Phi(t)(\Phi(r-T)-\Phi(r))^{-1} g\left(r, y_{r}\right) d r \\
& +\sum_{j: t_{j} \in[t, t+T)} \Phi(t)\left(\Phi\left(t_{j}-T\right)-\Phi\left(t_{j}\right)\right)^{-1} I_{j}\left(y\left(t_{j}\right)\right) .
\end{aligned}
$$

Consequently, let $y$ be a $T$-periodic solution of (2.2). If $t \neq t_{i}, i \in \mathbb{Z}$, then by (2.2), we have

$$
\begin{aligned}
y(t)= & \Phi(t)\left[\int_{t}^{t+T}(\Phi(r-T)-\Phi(r))^{-1} g\left(r, y_{r}\right) d r\right. \\
& \left.+\sum_{j: t_{j} \in[t, t+T)}\left(\Phi\left(t_{j}-T\right)-\Phi\left(t_{j}\right)\right)^{-1} I_{j}\left(y\left(t_{j}\right)\right)\right] .
\end{aligned}
$$

Therefore, by (2.1), we obtain

$$
\begin{aligned}
y^{\prime}(t)= & \Phi^{\prime}(t)\left[\int_{t}^{t+T}(\Phi(r-T)-\Phi(r))^{-1} g\left(r, y_{r}\right) d r\right. \\
& \left.+\sum_{j: t_{j} \in[t, t+T)}\left(\Phi\left(t_{j}-T\right)-\Phi\left(t_{j}\right)\right)^{-1} I_{j}\left(y\left(t_{j}\right)\right)\right] \\
& +\Phi(t)\left[(\Phi(t)-\Phi(t+T))^{-1} g\left(t+T, y_{t+T}\right)\right. \\
& \left.-(\Phi(t-T)-\Phi(t))^{-1} g\left(t, y_{t}\right)\right] \\
= & A(t) \Phi(t)\left[\int_{t}^{t+T}(\Phi(r-T)-\Phi(r))^{-1} g\left(r, y_{r}\right) d r\right. \\
& \left.+\sum_{j: t_{j} \in[t, t+T)}\left(\Phi\left(t_{j}-T\right)-\Phi\left(t_{j}\right)\right)^{-1} I_{j}\left(y\left(t_{j}\right)\right)\right] \\
& +\Phi(t)\left[(\Phi(t)-\Phi(t+T))^{-1}-(\Phi(t-T)-\Phi(t))^{-1}\right] g\left(t, y_{t}\right) \\
= & A(t) y(t)+g\left(t, y_{t}\right) .
\end{aligned}
$$

If $t=t_{i}, i \in \mathbb{Z}$, then by (2.1) and (2.2), we get

$$
\begin{aligned}
y\left(t_{i}^{+}\right)-y\left(t_{i}^{-}\right)= & \sum_{j: t_{j} \in\left[t_{i}^{+}, t_{i}^{+}+T\right)} \Phi\left(t_{i}\right)\left(\Phi\left(t_{j}-T\right)-\Phi\left(t_{j}\right)\right)^{-1} I_{j}\left(y\left(t_{j}\right)\right) \\
& -\sum_{j: t_{j} \in\left[t_{i}^{-}, t_{i}^{-}+T\right)} \Phi\left(t_{i}\right)\left(\Phi\left(t_{j}-T\right)-\Phi\left(t_{j}\right)\right)^{-1} I_{j}\left(y\left(t_{j}\right)\right) \\
& =\Phi\left(t_{i}\right)\left[\left(\Phi\left(t_{i}\right)-\Phi\left(t_{i}+T\right)\right)^{-1}-\left(\Phi\left(t_{i}-T\right)-\Phi\left(t_{i}\right)\right)^{-1}\right] I_{j}\left(y\left(t_{j}\right)\right) \\
& =I_{j}\left(y\left(t_{j}\right)\right) .
\end{aligned}
$$

So $y$ is also a $T$-periodic solution of (1.3). 
Define, for $i, k=1, \ldots, n$,

$$
A_{0}:=\min _{0 \leq t \leq u \leq T}\left|G_{i k}(t, u)\right|>0, \quad B_{0}:=\max _{0 \leq t \leq u \leq T}\left|G_{i k}(t, u)\right|>0
$$

and

$$
A:=\min _{0 \leq t \leq u \leq T}\left|\sum_{i=1}^{n} G_{i k}(t, u)\right|>0, \quad B:=\max _{0 \leq t \leq u \leq T}\left|\sum_{i=1}^{n} G_{i k}(t, u)\right|>0 .
$$

Let

$$
\begin{array}{r}
K=\left\{y(\cdot)=\left(y_{1}(\cdot), \ldots, y_{n}(\cdot)\right)^{T} \in X: y_{i}(t) \geq \lambda\left\|y_{i}\right\|, t \in[0, T],\right. \\
i=1, \ldots, n\},
\end{array}
$$

where $\lambda=A_{0} / B_{0} \in(0,1)$ and $A_{0}, B_{0}$ are defined above. Obviously, $K$ is a cone in $X$.

Define a mapping $H$ by

$$
(H y)(t)=\int_{t}^{t+T} G(t, r) g\left(r, y_{r}\right) d r+\sum_{j: t_{j} \in[t, t+T)} G\left(t, t_{j}\right) I_{j}\left(y\left(t_{j}\right)\right),
$$

for all $y \in K, t \in \mathbb{R}$, where $G(t, u)$ is defined by (2.3) and

$$
(H y)(t)=\left(\left(H_{1} y\right)(t), \ldots,\left(H_{n} y\right)(t)\right)^{T},
$$

where

$$
\left(H_{i} y\right)(t)=\int_{t}^{t+T} \sum_{k=1}^{n} G_{i k} g_{k}\left(r, y_{r}\right) d r+\sum_{j: t_{j} \in[t, t+T)} \sum_{k=1}^{n} G_{i k} I_{j}^{k}\left(y\left(t_{j}\right)\right), \quad i=1, \ldots, n .
$$

Now we claim that

$$
G(t+T, u+T)=G(t, u) .
$$

In fact,

$$
\begin{aligned}
G(t+T, u+T) & =\Phi(t+T)(\Phi(u)-\Phi(u+T))^{-1} \\
& =\Phi(t) e^{B T}\left(\Phi(u)-\Phi(u) e^{B T}\right)^{-1} \\
& =\Phi(t)\left(\Phi(u) e^{-B T}-\Phi(u)\right)^{-1} \\
& =\Phi(t)(\Phi(u-T)-\Phi(u))^{-1}=G(t, u) .
\end{aligned}
$$

Hereafter, we always assume that

$$
\left(H_{3}\right) g_{k} G_{i k}>0 \text { and } I_{j}^{k} G_{i k}>0 \text {, for all } i, k=1, \ldots, n, j \in \mathbb{Z} .
$$

Then we can easily get

$$
g_{k} \sum_{i=1}^{n} G_{i k}>0, \quad I_{j}^{k} \sum_{i=1}^{n} G_{i k}>0, \quad \forall k=1, \ldots, n, j \in \mathbb{Z} .
$$

We now give some lemmas concerning $K$ and $H$ defined by (2.6) and (2.7), respectively. 
Lemma 2.4. Assume that $\left(H_{3}\right)$ holds. Then $H: K \rightarrow K$ is well defined.

Proof. For any $y \in K$, it is clear that $H y \in P C\left(\mathbb{R}, \mathbb{R}^{n}\right)$. In view of $(2.8)$ we obtain

$$
\begin{aligned}
(H y)(t+T)= & \int_{t+T}^{t+2 T} \Phi(t+T)(\Phi(r-T)-\Phi(r))^{-1} g\left(r, y_{r}\right) d r \\
& +\sum_{j: t_{j} \in[t+T, t+2 T)} \Phi(t+T)\left(\Phi\left(t_{j}-T\right)-\Phi\left(t_{j}\right)\right)^{-1} I_{j}\left(y\left(t_{j}\right)\right) \\
= & \int_{t}^{t+T} \Phi(t+T)(\Phi(s)-\Phi(s+T))^{-1} g\left(s+T, y_{s+T}\right) d s \\
& +\sum_{i: t_{i} \in[t, t+T)} \Phi(t+T)\left(\Phi\left(t_{i}\right)-\Phi\left(t_{i}+T\right)\right)^{-1} I_{i}\left(y\left(t_{i}\right)\right) \\
= & \int_{t}^{t+T} G(t+T, s+T) g\left(s, y_{s}\right) d s \\
& +\sum_{i: t_{i} \in[t, t+T)} G\left(t+T, t_{i}+T\right) I_{i}\left(y\left(t_{i}\right)\right) \\
= & \int_{t}^{t+T} G(t, s) g\left(s, y_{s}\right) d s+\sum_{i: t_{i} \in[t, t+T)} G\left(t, t_{i}\right) I_{i}\left(y\left(t_{i}\right)\right)=(H y)(t) .
\end{aligned}
$$

That is,

$$
(H y)(t+T)=(H y)(t), \quad t \in \mathbb{R} .
$$

So $H y \in X$.

For any $y \in K$ and $t \in[0, T]$, we have

$$
\begin{aligned}
\left|H_{i} y\right| & =\left|\int_{t}^{t+T} \sum_{k=1}^{n} G_{i k} g_{k} d r+\sum_{j: t_{j} \in[t, t+T)} \sum_{k=1}^{n} G_{i k} I_{j}^{k}\right| \\
& \leq \int_{t}^{t+T} \sum_{k=1}^{n}\left|G_{i k}\right|\left|g_{k}\right| d r+\sum_{j=1}^{p} \sum_{k=1}^{n}\left|G_{i k}\right|\left|I_{j}^{k}\right| \\
& \leq B_{0}\left(\int_{t}^{t+T} \sum_{k=1}^{n}\left|g_{k}\right| d r+\sum_{j=1}^{p} \sum_{k=1}^{n}\left|I_{j}^{k}\right|\right), \quad i=1, \ldots, n .
\end{aligned}
$$

So

$$
\left\|H_{i} y\right\|=\sup _{t \in[0, T]}\left|H_{i} y\right| \leq B_{0}\left(\int_{t}^{t+T} \sum_{k=1}^{n}\left|g_{k}\right| d r+\sum_{j=1}^{p} \sum_{k=1}^{n}\left|I_{j}^{k}\right|\right), \quad i=1, \ldots, n,
$$


and by $\left(H_{3}\right)$, we get

$$
\begin{aligned}
\left(H_{i} y\right)(t) & =\int_{t}^{t+T} \sum_{k=1}^{n}\left|G_{i k}\right|\left|g_{k}\right| d r+\sum_{j: t_{j} \in[t, t+T)} \sum_{k=1}^{n}\left|G_{i k}\right|\left|I_{j}^{k}\right| \\
& \geq A_{0}\left(\int_{t}^{t+T} \sum_{k=1}^{n}\left|g_{k}\right| d r+\sum_{j=1}^{p} \sum_{k=1}^{n}\left|I_{j}^{k}\right|\right) \\
& =\frac{A_{0}}{B_{0}} B_{0}\left(\int_{t}^{t+T} \sum_{k=1}^{n}\left|g_{k}\right| d r+\sum_{j=1}^{p} \sum_{k=1}^{n}\left|I_{j}^{k}\right|\right) \geq \lambda\left\|H_{i} y\right\|, \quad i=1, \ldots, n,
\end{aligned}
$$

i.e., $H y \in K$. This completes the proof.

Lemma 2.5. Assume that $\left(H_{1}\right)$ and $\left(H_{2}\right)$ hold. Then $H: K \rightarrow K$ is completely continuous.

Proof. We first show that $H$ is continuous. By $\left(H_{2}\right)$, for any $L>0$ and $\varepsilon>0$, there exists a $\delta>0$ such that $\left[\phi, \varphi \in B C\left(\mathbb{R}, \mathbb{R}^{n}\right),\|\phi\| \leq L,\|\varphi\| \leq L\right.$, $\|\phi-\varphi\|<\delta]$ implies

$$
\sup _{0 \leq s \leq T}\left|g\left(s, \phi_{s}\right)-g\left(s, \varphi_{s}\right)\right|_{0}<\frac{\varepsilon}{2 B T} .
$$

And since $I_{j} \in C\left(\mathbb{R}^{n}, \mathbb{R}^{n}\right)$, we have

$$
\left|I_{j}(\phi)-I_{j}(\varphi)\right|_{0}<\frac{\varepsilon}{2 B p}, \quad j \in \mathbb{Z} .
$$

If $x, y \in K$ with $\|x\| \leq L,\|y\| \leq L$ and $\|x-y\|<\delta$, then

$$
\begin{aligned}
|(H x)(t)-(H y)(t)|_{0} & =\sum_{i=1}^{n} \mid \int_{t}^{t+T} \sum_{k=1}^{n} G_{i k} g_{k}\left(r, x_{r}\right) d r+\sum_{j: t_{j} \in[t, t+T)} \sum_{k=1}^{n} G_{i k} I_{j}^{k}\left(x\left(t_{j}\right)\right) \\
& \quad-\int_{t}^{t+T} \sum_{k=1}^{n} G_{i k} g_{k}\left(r, y_{r}\right) d r-\sum_{j: t_{j} \in[t, t+T)} \sum_{k=1}^{n} G_{i k} I_{j}^{k}\left(y\left(t_{j}\right)\right) \mid \\
\leq & \int_{t}^{t+T} \sum_{k=1}^{n}\left|\sum_{i=1}^{n} G_{i k}\right|\left|g_{k}\left(r, x_{r}\right)-g_{k}\left(r, y_{r}\right)\right| d r \\
& +\sum_{j: t_{j} \in[t, t+T)} \sum_{k=1}^{n}\left|\sum_{i=1}^{n} G_{i k}\right|\left|I_{j}^{k}\left(x\left(t_{j}\right)\right)-I_{j}^{k}\left(y\left(t_{j}\right)\right)\right|
\end{aligned}
$$




$$
\begin{aligned}
& \leq B\left(\int_{0}^{T}\left|g\left(s, x_{s}\right)-g\left(s, y_{s}\right)\right|_{0} d s+\sum_{j=1}^{p}\left|I_{j}(x)-I_{j}(y)\right|_{0}\right) \\
& \leq B\left(T \frac{\varepsilon}{2 B T}+p \frac{\varepsilon}{2 B p}\right)=\varepsilon
\end{aligned}
$$

for all $t \in[0, T]$. This yields $\|H x-H y\|<\varepsilon$. Thus $H$ is continuous.

Next, we show that $H$ maps bounded sets in $K$ into relatively compact sets. We first prove that $g$ maps bounded sets into bounded sets. Indeed, let $\varepsilon=\min \{2 B T, 2 B p\}$. By $\left(H_{2}\right)$ and continuity of $I_{j}$, for any $\mu>0$, there exists $\delta>0$ such that for $x, y \in B C\left(\mathbb{R}, \mathbb{R}^{n}\right)$, the inequalities $\|x\| \leq L$, $\|y\| \leq L,\|x-y\|<\delta$ imply

$$
\sup _{0 \leq s \leq T}\left|g\left(s, x_{s}\right)-g\left(s, y_{s}\right)\right|_{0}<1
$$

and

$$
\left|I_{j}(x)-I_{j}(y)\right|_{0}<1, \quad j \in \mathbb{Z} .
$$

Choose a positive integer $N$ such that $\mu / N<\delta$. Let $y \in B C\left(\mathbb{R}, \mathbb{R}^{n}\right)$ and define $y^{k}(t)=k y(t) / N$ for $k=0,1, \ldots, N$. If $\|y\| \leq \mu$ then

$$
\left\|y^{k}-y^{k-1}\right\|=\sup _{t \in[0, T]}\left|y(t) \frac{k}{N}-y(t) \frac{k-1}{N}\right|_{0} \leq\|y\| \frac{1}{N} \leq \frac{\mu}{N}<\delta .
$$

Thus,

$$
\left|g\left(s, y_{s}^{k}\right)-g\left(s, y_{s}^{k-1}\right)\right|_{0}<1
$$

and

$$
\left|I_{j}\left(y^{k}\left(t_{j}\right)\right)-I_{j}\left(y^{k-1}\left(t_{j}\right)\right)\right|_{0}<1, \quad j \in \mathbb{Z},
$$

for all $s, t_{j} \in[0, T]$. This yields

$$
\begin{aligned}
\left|g\left(s, y_{s}\right)\right|_{0} & =\left|g\left(s, y_{s}^{N}\right)\right|_{0} \leq \sum_{k=1}^{N}\left|g\left(s, y_{s}^{k}\right)-g\left(s, y_{s}^{k-1}\right)\right|_{0}+|g(s, 0)|_{0} \\
& <N+|g(s, 0)|_{0}=: Q
\end{aligned}
$$

and

$$
\begin{aligned}
\left|I_{j}\left(y\left(t_{j}\right)\right)\right|_{0}=\left|I_{j}\left(y^{N}\left(t_{j}\right)\right)\right|_{0} & \leq \sum_{k=1}^{N}\left|I_{j}\left(y^{k}\left(t_{j}\right)\right)-I_{j}\left(y^{k-1}\left(t_{j}\right)\right)\right|_{0}+\left|I_{j}(0)\right|_{0} \\
& <N+\left|I_{j}(0)\right|_{0}=: R, \quad j \in \mathbb{Z} .
\end{aligned}
$$

It follows from (2.6) that

$$
\begin{aligned}
\|H y\|=\sup _{t \in[0, T]} \sum_{i=1}^{n}\left|\left(H_{i} y\right)(t)\right| & \leq \sum_{k=1}^{n} B\left(\int_{0}^{T}\left|g_{k}\right| d s+\sum_{j=1}^{p}\left|I_{j}^{k}\right|\right) \\
& =B\left(|g|_{0} T+p\left|I_{j}\right|_{0}\right) \leq B(Q T+p R)=: M .
\end{aligned}
$$


Finally, for $t \in \mathbb{R}$, we have

$$
\frac{d}{d t}[(H y)(t)]=A(t)(H y)(t)+g\left(t, y_{t}\right) .
$$

So

$$
\left|\frac{d}{d t}[(H y)(t)]\right|_{0} \leq|A(t)(H y)(t)|_{0}+\left|g\left(t, y_{t}\right)\right|_{0} \leq|A| M+Q,
$$

where $|A|=\max _{1 \leq i \leq n} \sup _{t \in[0, T]} \sum_{j=1}^{n}\left|a_{i j}(t)\right|$.

Hence $\{H y: y \in K,\|y\| \leq \mu\}$ is a family of uniformly bounded and equi-continuous functions on $[0, T]$. By the theorem of Ascoli-Arzelà, the operator $H$ is completely continuous.

3. Main result. Our main result is as follows:

TheOREM 3.1. Assume that $\left(H_{1}\right)$ and $\left(H_{2}\right)$ are satisfied and there exist $a, b>0$ with $a<b$ such that

(i) $g^{0}+I^{0}<1 / B$, and $g^{\infty}+I^{\infty}<1 / B$;

(ii) $\check{g}+\check{I}>\delta b / A$ for $\delta b \leq\|u\| \leq b, t \in \mathbb{R}$.

Then system (1.3) has at least three positive T-periodic solutions.

Proof. By the condition $g^{\infty}+I^{\infty}<1 / B$ of (i), we deduce that for

$$
0<\varepsilon<\frac{1 / B-\left(g^{\infty}+I^{\infty}\right)}{2},
$$

there exists a $C_{0}$ such that

$$
\int_{0}^{T}\left|g\left(s, y_{s}\right)\right|_{0} d s \leq\left(g^{\infty}+\varepsilon\right)\|y\|, \quad \sum_{j=1}^{p}\left|I_{j}(y)\right|_{0} \leq\left(I^{\infty}+\varepsilon\right)\|y\|,
$$

where $y>C_{0}$.

Let $C_{1}=C_{0} / \delta$, where $\delta=A / B$. If $y \in K$ and $\|y\|>C_{1}$, then $y>C_{0}$ and we have

$$
\begin{aligned}
|(H y)(t)|_{0} & \leq B \sum_{k=1}^{n} \int_{0}^{T}\left|g_{k}\left(s, y_{s}\right)\right| d s+B \sum_{k=1}^{n} \sum_{j=1}^{p}\left|I_{j}^{k}\left(y\left(t_{j}\right)\right)\right| \\
& =B \int_{0}^{T}\left|g\left(s, y_{s}\right)\right|_{0} d s+B \sum_{j=1}^{p}\left|I_{j}\left(y\left(t_{j}\right)\right)\right|_{0} \\
& \leq B\left(g^{\infty}+I^{\infty}+2 \varepsilon\right)\|y\| \\
& <B\left[g^{\infty}+I^{\infty}+\left(1 / B-\left(g^{\infty}+I^{\infty}\right)\right)\right]\|y\|=\|y\| .
\end{aligned}
$$

Take $K_{C_{1}}=\left\{y \in K:\|y\| \leq C_{1}\right\}$. Then $K_{C_{1}}$ is a bounded set. Since $H$ is completely continuous, it maps bounded sets into bounded sets and there 
exists a number $C_{2}$ such that

$$
\|H y\| \leq C_{2}, \quad \forall y \in K_{C_{1}} .
$$

If $C_{2} \leq C_{1}$, we deduce that $H: K_{C_{1}} \rightarrow K_{C_{1}}$ is completely continuous. If $C_{1}<C_{2}$, then from (3.1), we know that for any $y \in K_{C_{2}} \backslash K_{C_{1}},\|y\|>C_{1}$ and $\|H y\|<\|y\|<C_{2}$ hold. Thus we see that $H: K_{C_{2}} \rightarrow K_{C_{2}}$ is completely continuous. Now, take $c=\max \left\{C_{1}, C_{2}\right\}$; obviously $c>b$, so $H: K_{c} \rightarrow K_{c}$ is completely continuous.

Define a positive continuous concave functional by $\alpha(y)=\inf _{t \in[0, T]}|y(t)|_{0}$. First, we let $a=\delta b$ and take $y \equiv(a+b) / 2$. Then $y \in K(\alpha, a, b), \alpha(y)>a$, and so $\{y \in K(\alpha, a, b)\} \neq \emptyset$. By (ii), if $y \in K(\alpha, a, b)$, then $\alpha(y)>a$, and we have

$$
\begin{aligned}
\alpha(H y)=\inf _{t \in[0, T]}|(H y)(t)|_{0} & \geq \inf _{t \in[0, T]}\left\{A \int_{t}^{t+T}\left|g\left(s, y_{s}\right)\right|_{0} d s+A \sum_{j=1}^{p}\left|I_{j}\left(y\left(t_{j}\right)\right)\right|_{0}\right\} \\
& \geq A(\check{g}+\check{I}) \geq A \frac{\delta b}{A}=a .
\end{aligned}
$$

Hence condition (i) of Lemma 2.1 holds.

Secondly, by the $g^{0}+I^{0}<1 / B$ condition of (i), one can find that for

$$
0<\varepsilon<\frac{1 / B-\left(g^{0}+I^{0}\right)}{2},
$$

there exists $0<d<a$ such that

$$
\int_{0}^{T}\left|g\left(s, y_{s}\right)\right|_{0} d s \leq\left(g^{0}+\varepsilon\right)\|y\|, \quad \sum_{j=1}^{p}\left|I_{j}\left(y\left(t_{j}\right)\right)\right|_{0} \leq\left(I^{0}+\varepsilon\right)\|y\|,
$$

where $0 \leq y \leq d$. If $y \in K_{d}=\{y:\|y\| \leq d\}$, we have

$$
\begin{aligned}
|(H y)(t)|_{0} & \leq B\left(\sum_{k=1}^{n} \int_{0}^{T}\left|g_{k}\left(s, y_{s}\right)\right| d s+\sum_{k=1}^{n} \sum_{j=1}^{p}\left|I_{j}^{k}\left(y\left(t_{j}\right)\right)\right|\right) \\
& =B\left(\int_{0}^{T}\left|g\left(s, y_{s}\right)\right|_{0} d s+\sum_{j=1}^{p}\left|I_{j}\left(y\left(t_{j}\right)\right)\right|_{0}\right) \\
& <B\left(g^{0}+I^{0}+2 \varepsilon\right)\|y\| \\
& <B\left(g^{0}+I^{0}+\left(1 / B-\left(g^{0}+I^{0}\right)\right)\right)\|y\| \\
& =\|y\| \leq d .
\end{aligned}
$$

That is, condition (ii) of Lemma 2.1 holds.

Finally, if $y \in K(\alpha, a, c)$ with $\|H y\|>b$, then by (3.1), we have

$$
b<\|H y\| \leq B\left(\int_{0}^{T}\left|g\left(s, y_{s}\right)\right|_{0} d s+\sum_{j=1}^{p}\left|I_{j}\left(y\left(t_{j}\right)\right)\right|_{0}\right),
$$


which implies that

$$
\begin{aligned}
\alpha(H y) & =\inf _{t \in[0, T]}|(H y)(t)|_{0} \geq A\left(\int_{0}^{T}\left|g\left(s, y_{s}\right)\right|_{0} d s+\sum_{j=1}^{p}\left|I_{j}\left(y\left(t_{j}\right)\right)\right|_{0}\right) \\
& >A \frac{b}{B}=\delta b=a,
\end{aligned}
$$

which means that condition (iii) of Lemma 2.1 holds.

Therefore, by Lemma 2.1, the operator $H$ has at least three fixed points $x_{1}, x_{2}, x_{3} \in \bar{K}_{c}$ such that

$x_{1} \in K_{d}, \quad x_{2} \in\{x \in K(\alpha, a, c), \alpha(x)>a\}, \quad x_{3} \in \bar{K}_{c} \backslash \alpha\left(K(\alpha, a, c) \cup \bar{K}_{d}\right)$. The proof of Theorem 3.1 is complete.

REMARK 3.1. From the proof of Theorem 3.1, one can easily see that if condition $(i)$ is replaced by

$$
\left(\mathrm{i}^{\prime}\right) \text { if } g^{0}=I^{0}=0 \text {, and } g^{\infty}=I^{\infty}=0,
$$

then the conclusion of Theorem 3.1 remains valid.

Acknowledgements. This work is supported by the National Natural Sciences Foundation of People's Republic of China under Grant 10971183 and the Natural Sciences Foundation of Yunnan Province.

\section{References}

[1] M. N. Islam and Y. N. Raffoul, Periodic solutions of neutral nonlinear system of differential equations with functional delay, J. Math. Anal. Appl. 331 (2007), 11751186.

[2] D. Jiang, J. Wei and B. Zhang, Positive periodic solutions of functional differential equations and population models, Electron. J. Differential Equations 2002, no. 71, $13 \mathrm{pp}$.

[3] R. W. Leggett and L. R. Williams, Multiple positive fixed points of nonlinear operators on ordered Banach spaces, Indiana Univ. Math. J. 28 (1979), 673-688.

[4] X. Li, X. Lin, D. Jiang and X. Zhang, Existence and multiplicity of positive periodic solutions to functional differential equations with impulse effects, Nonlinear Anal. 62 (2005), 683-701.

[5] X. Li, X. Zhang and D. Jiang, A new existence theory for positive periodic solutions to functional differential equations with impulse effects, Comput. Math. Appl. 51 (2006), 1761-1772.

[6] Y. K. Li, Positive periodic solutions of nonlinear differential systems with impulses, Nonlinear Anal. 68 (2008), 2389-2405.

[7] Y. K. Li and Z. W. Xing, Existence and global exponential stability of periodic solution of CNNs with impulses, Chaos Solitons Fractals 33 (2007), 1686-1693.

[8] Y. K. Li and L. Zhu, Positive periodic solutions of nonlinear functional differential equations, Appl. Math. Comput. 156 (2004), 329-339.

[9] P. De Nápoli and M. C. Mariani, Three solutions for quasilinear equations in $\mathbb{R}^{n}$ near resonance, Electron. J. Differential Equations Conf. 6 (2001), 131-140. 
[10] J. J. Nieto, Basic theory for nonresonance impulsive periodic problems of first order, J. Math. Anal. Appl. 205 (1997), 423-433.

[11] H. Sun and Y. Wang, Existence of positive solutions for p-Laplacian three-point boundary-value problems on time scales, Electron. J. Differential Equations 2008, no. $92,14 \mathrm{pp}$.

[12] W. Walter, Ordinary Differential Equations, Springer, New York, 1991.

[13] N. Zhang, B. Dai and X. Qian, Periodic solutions for a class of higher-dimension functional differential equations with impulses, Nonlinear Anal. 68 (2008), 629-638.

[14] X. Zhang, J. Yan and A. Zhao, Existence of positive periodic solutions for an impulsive differential equation, ibid. 68 (2008), 3209-3216.

[15] J. W. Zhou and Y. K. Li, Existence and multiplicity of solutions for some Dirichlet problems with impulsive effects, ibid. 71 (2009), 2856-2865.

Yongkun Li (corresponding author), Changzhao Li, Juan Zhang

Department of Mathematics

Yunnan University

Kunming, Yunnan 650091, People's Republic of China

E-mail: yklie@ynu.edu.cn

Received 6.4.2009

and in final form 2.9.2009

$(2001)$ 
